\title{
Photoinduced Absorption and Pulsed Recording of Dynamic Holograms in Bismuth Silicate Crystals
}

\author{
I. G. Dadenkov ${ }^{a}$, A. L. Tolstik ${ }^{a, *}$, Yu. I. Miksyuk ${ }^{b}$, and K. A. Saechnikov ${ }^{b}$ \\ ${ }^{a}$ Belarusian State University, Minsk, 220030 Belarus \\ ${ }^{b}$ Belarusian State Pedagogical University, Minsk, 220030 Belarus \\ *e-mail:Tolstik@bsu.by
}

Received March 15, 2020; revised March 15, 2020; accepted May 20, 2020

\begin{abstract}
The dynamics of photoinduced absorption and holographic-grating recording in photorefractive crystals of bismuth silicate is studied. It is shown that, with the use of nanosecond laser pulses and of the intensity on the order of $1 \mathrm{MW} / \mathrm{cm}^{2}$ or higher, the induced absorption due to population of the short-lived trapping levels, with characteristic relaxation times about several milliseconds or tens of milliseconds, is the case. Recording of dynamic holograms has been realized in these conditions in bismuth silicate crystals. Two mechanisms of holographic-grating recording, with the lifetimes differing by three orders of magnitude, are established. At relatively low intensities, about $1 \mathrm{MW} / \mathrm{cm}^{2}$ or lower, the medium response is determined by a photorefractive mechanism of nonlinearity, with relaxation times of several seconds. At the intensities exceeding $5 \mathrm{MW} / \mathrm{cm}^{2}$, one can observe a fast (ms relaxation times) component that may be associated with population of the short-lived traps. It is shown that the contribution of each mechanism is greatly dependent on the intensity of laser radiation and, for the intensities above $10-15 \mathrm{MW} / \mathrm{cm}^{2}$, the short-lived traps having millisecond lifetimes play the decisive role.
\end{abstract}

Keywords: holography, dynamic holograms, photoinduced absorption, sillenites, bismuth silicate

DOI: $10.1134 /$ S0030400X20090052

\section{INTRODUCTION}

One of the significant advantages of cubic photorefractive crystals from the sillenites family $\left(\mathrm{Bi}_{12} \mathrm{SiO}_{20}\right.$, $\mathrm{Bi}_{12} \mathrm{TiO}_{20}, \mathrm{Bi}_{12} \mathrm{GeO}_{20}$ ) is the real-time formation of dynamic holograms. This property enables the use of these crystals in adaptive interferometers, in systems of associative memory, optical image amplification, holographic recording, data storage and processing, and the like [1-6]. The physical processes of hologram recording in photorefractive crystals are based on spatial redistribution of the charges in a field of interfering light beams over the numerous centers having different nature and characteristics.

By their structure, photorefractive crystals are classified with wide-band dielectrics. Of great importance are impurities and structural defects of the crystal lattice, which lead to the formation of donor and acceptor energy levels in the forbidden band [5, 7]. A distinctive feature is the fact that both the long-lived (seconds, hours) and short-lived (micro- and milliseconds) traps can exist simultaneously [8-13]. The transitions from a level in the forbidden band to the conduction band result in the formation of mobile charge carriers. It should be noted that such transitions are feasible when using radiation with a considerably longer wavelength than in the case of a direct band-to-band transition, offering the possibility of operations in the visible and near IR spectral regions $[5,14]$. Due to the subsequent diffusion or electron drift in an external electric field, the trapping levels become populated, a field of the space charge is formed, and the refractive index is modulated in consequence of the Pockels effect. In this way a holographic grating is formed, with the dynamics governed by that of the spatial charge field. As a rule, dynamic holograms are formed in refractive crystals by means of continuous laser radiation [1-4, 6, 7]. The use of nano- and pico-second pulses offers new possibilities [15-18].

This paper presents the results obtained during a study of the processes of photoinduced absorption and of the short- or long-lived holographic grating formation in photorefractive crystals of bismuth silicate (BSO), characterized by a complex structure of the defect centers, in conditions of pulsed laser excitation.

\section{Photoinduced Absorption}

The photoinduced absorption in crystals from the sillenites family in the visible and near IR regions is determined by population of the trapping levels lying in the forbidden band of a crystal. The presence of such levels in sillenite crystals is associated with hardly 


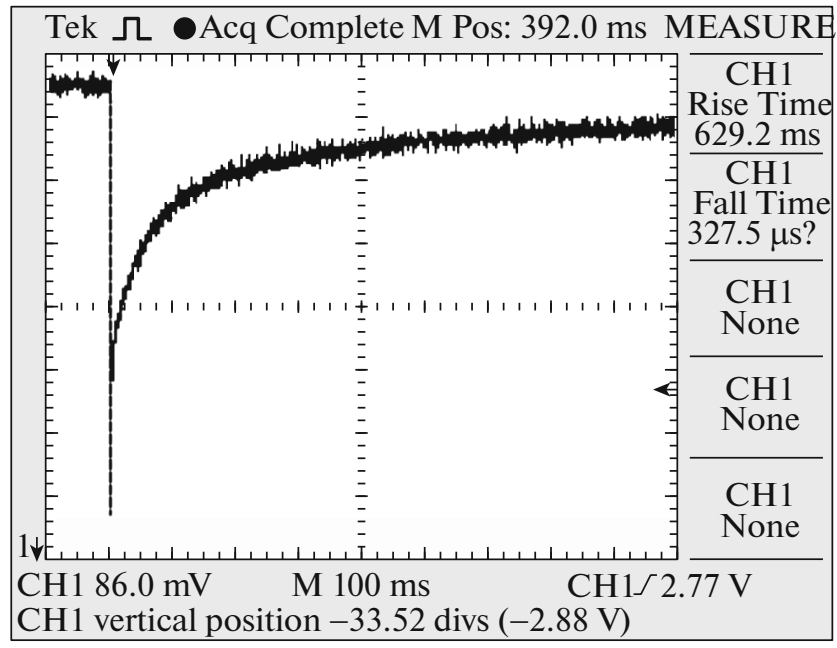

Fig. 1. Characteristic oscillogram for radiation transmitted through a crystal of BSO subjected to the effect of a laser pulse with the intensity $5 \mathrm{MW} / \mathrm{cm}^{2}$.

controlled impurities and defects, which have a considerable influence on the position and the occupation probabilities of short- and long-lived traps. Owing to investigation of the induced absorption dynamics, we can analyze the processes proceeding in the crystals under the effect of pulsed laser radiation. Figure 1 shows a typical oscillogram for radiation transmitted through a bismuth silicate crystal at the wavelength $632.8 \mathrm{~nm}$ (helium-neon laser) after the action of a 20 -ns laser pulse at the wavelength $532 \mathrm{~nm}$. It is seen that the intensity is lowered considerably for the times of several hundred milliseconds. We can discern the stage of the fast $(\sim 10 \mathrm{~ms})$ decreasing induced absorption and the stage of slow relaxation $(>100 \mathrm{~ms})$.

The processes taking place in a crystal of bismuth silicate under pulsed laser irradiation are illustrated in Fig. 2 showing a diagram for the energy states and the photoexcitation and relaxation processes [19]. The induced absorption is determined by the electron transitions from valence band 1 to conduction band 4 , with subsequent recombination into short-lived 3 and longlived 2 energy levels (traps) positioned in the forbidden band. A width of the forbidden band for a bismuth silicate crystal comes to $3.08 \mathrm{eV}$, preventing realization of a direct band-to-band transition for photon absorption at the wavelength $532 \mathrm{~nm}$ (quantum energy $2.33 \mathrm{eV}$ ) used during the experiments. To explain absorption of high-power laser pulses, we take into consideration a mechanism of two-quantum light absorption, the transition rates for which are proportional to the squared intensity of incident radiation.

The photoinduced absorption is described by a system of equations derived considering single-photon absorption by the electrons from trapping levels and

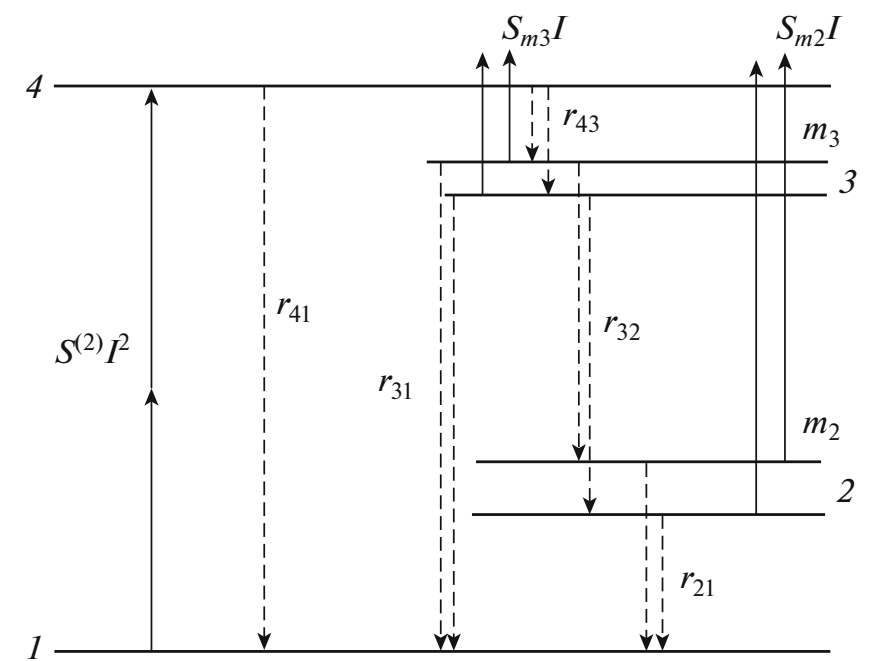

Fig. 2. The energy-level diagram and the photoexcitation, recombination, and mobile-electron trapping processes in a bismuth silicate crystal.

two-quantum transitions from the valence to the forbidden band [19]:

$$
\begin{gathered}
\frac{\partial n}{\partial t}=\frac{\gamma}{2 \hbar} I_{0}^{2}+m_{3} S_{m 3} I+m_{2} S_{m 2} I \\
-r_{41} n p-r_{43} n\left(M_{3}-m_{3}\right), \\
\frac{\partial m_{3}}{\partial t}=-m_{3} S_{m 3} I+r_{43} n\left(M_{3}-m_{3}\right) \\
-r_{32} m_{3}\left(M_{32}-m_{2}\right)-r_{31} m_{3} p, \\
\frac{\partial m_{2}}{\partial t}=-m_{2} S_{m 2} I+r_{32} m_{3}\left(M_{2}-m_{2}\right)-r_{21} m_{2},
\end{gathered}
$$

where $n$-electron concentration in the conduction band, $m_{2}$ and $m_{3}$-electron concentration at a longlived level and a short-lived level, respectively; $M_{2}$ and $M_{3}$-total concentrations of the long- and short-lived levels, $p$-hole concentration in the valence band, $\gamma-$ two-quanta absorption factor, $S_{m 2}$ and $S_{m 3}$-photoionization cross-sections for the corresponding trapping levels, $r_{i j}$-recombination coefficients.

Considering the charge conservation condition, the number of holes in the valence band equals the total quantity of electrons in the conduction band and at the trapping levels

$$
p=n+m_{2}+m_{3} .
$$

With regard to a great spread in the rates of transitions between different states with the characteristic lifetimes ranging from a few microseconds to several tens of hours, it seems expedient to select some stages for convenient analysis of the population and relaxation processes of trapping levels.

The first stage is associated with excitation. Absorbing the light quanta, electrons leave the valence band and trapping levels for the conduction band- 
transitions 1-4, 2-4, 3-4 in Fig. 2. System of equations (1)-(3) is transformed as follows:

$$
\begin{aligned}
\frac{n}{\partial t}=\gamma 2 \hbar I_{0}^{2} & +m_{3} S_{m 3} I_{0}+m_{2} S_{m 2} I_{0}, \\
\frac{m_{3}}{\partial t} & =-m_{3} S_{m 3} I_{0}, \\
\frac{\partial m_{2}}{\partial t} & =-m_{2} S_{m 2} I_{0} .
\end{aligned}
$$

As the recombination rate is high, the lifetime of electrons in the conduction band is very short. As a result, electrons return to the valence band or go to the short-lived level 3 lying close to the conduction band. These transitions may be considered the second stage (nano- or microsecond relaxation) and are given by the equations

$$
\begin{gathered}
\frac{\partial n}{\partial t}=-r_{41} n p-r_{43} n\left(M_{3}-m_{3}\right), \\
\frac{\partial m_{3}}{\partial t}=r_{43} n\left(M_{3}-m_{3}\right) .
\end{gathered}
$$

The third stage taking several milliseconds represents relaxation associated with transition 3-1:

$$
\frac{\partial m_{3}}{\partial t}=-r_{31} m_{3} p .
$$

In the process, a part of electrons is trapped by the long-lived traps (transition 3-2). The probability of such a process is lower but it allows for population of long-lived levels 2, with the lifetimes making several tens of hours, when a large numbers of laser pulses are used [9].

Next, consider equation (10), the solution of which, in the assumption of the lowly populated longlived traps $\left(m_{2}=0\right.$ and $\left.m_{3}=p\right)$, is of the form

$$
m_{3}=\frac{m_{30}}{1+t / \tau},
$$

where $m_{30}$-initial electron concentration at a trapping level, $\tau$-characteristic lifetime that is inversely proportional to the recombination coefficient $r_{31}$.

However, this relation is at variance with the experimental data obtained at short lifetimes (below $10 \mathrm{~ms}$ ). Taking into account a great number of different impurities and defect centers characteristic for bismuth silicate crystals, in an effort to explain the observed divergences, we divide trapping level 3 into two sublevels $3 a$ and $3 b$ with different lifetimes. For the sublevel with a shorter lifetime, $3 a$, a solution for equation (5), in the conditions of marked population of long-lived level $3 b$, may be given as

$$
m_{3 a}=m_{3 a 0} \exp \left(-t / \tau_{3 a}\right) .
$$

For the sublevel with a longer lifetime, we have

$$
m_{3 b}=\frac{m_{3} b 0}{1+t / \tau_{b}} .
$$

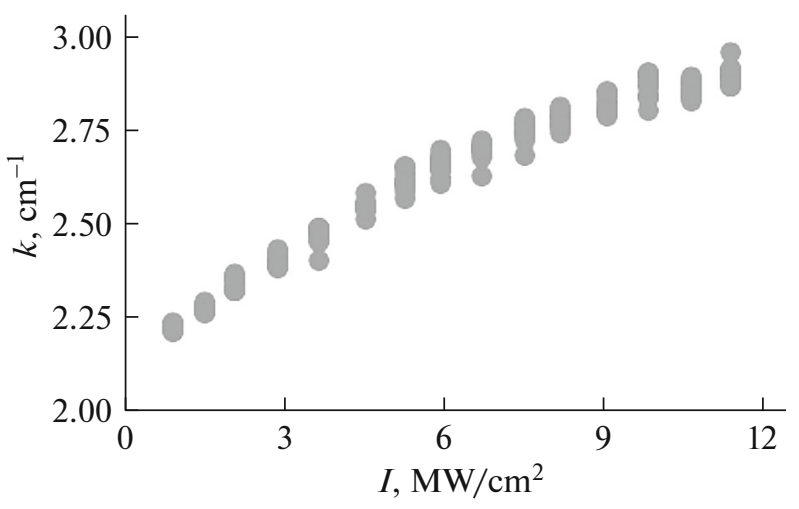

Fig. 3. The absorption factor as a function of the pulse radiation intensity for a crystal of BSO.

Based on equations (12), (13), the absorption factor associated with population of the indicated sublevels is of the form

$$
k=k_{3 a 0} \exp \left(-\frac{t}{\tau_{3 a}}\right)+\frac{k_{3 b 0}}{1+t / \tau_{3 b}},
$$

where $k_{3 a 0}, k_{3 b 0}$-initial absorption factors associated with the corresponding sublevels, $\tau_{3 a}$ and $\tau_{3 b}$-their lifetimes.

As demonstrated by a quantitative analysis of the recorded oscillograms, the above expression gives an adequate description for the experimental results and hence may be used to find the characteristic relaxation times.

Figure 3 presents the absorption factor as a function of the incident radiation intensity. As seen, the photoinduced absorption is effectively exhibited for the intensities above $1 \mathrm{MW} / \mathrm{cm}^{2}$, whereas at the intensities exceeding $10 \mathrm{MW} / \mathrm{cm}^{2}$ an increase of the induced absorption becomes saturated. The characteristic relaxation times are found by means formula (14). Figure 4 shows the curves for the relaxation time of trapping sublevels as a function of the laser radiation intensity. As the intensity is growing, the relaxation time decreases and has a constant value at the intensities above $5 \mathrm{MW} / \mathrm{cm}^{2}$. As this takes place, times of fast relaxation processes amount to several milliseconds, while relaxation of slow processes takes about $50 \mathrm{~ms}$.

\section{Pulse Recording of Dynamic Holograms}

The conducted studies of photoinduced absorption in bismuth silicate crystals have enabled the determination of conditions for recording of dynamic holograms on pulsed laser excitation. The population and relaxation dynamics of holographic gratings has been studied using the experimental setup shown in Fig. 5.

The experiments have been realized using the second-harmonic radiation of a pulsed neodymium- 


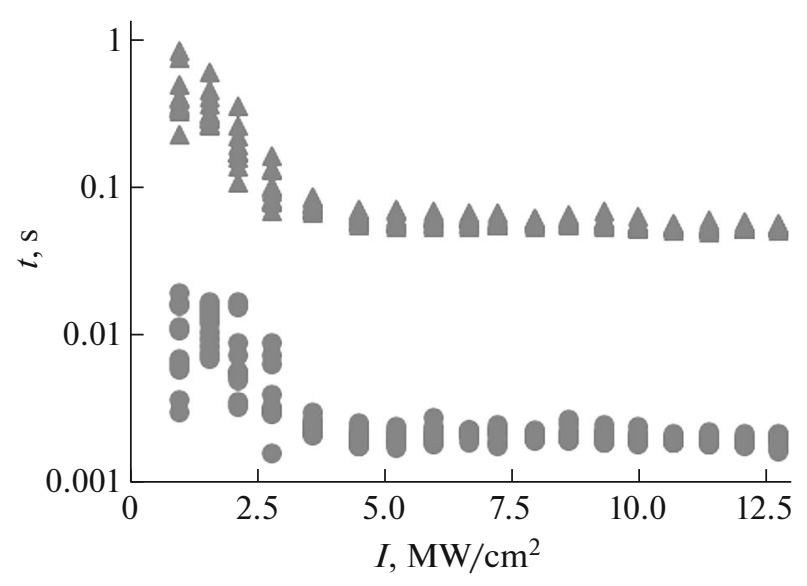

Fig. 4. The relaxation time of trapping sublevels as a function of the incident radiation intensity.

doped yttrium aluminum garnet laser $(\lambda=532 \mathrm{~nm}$ and 20 -ns pulse length), a low-power continuously operated helium-neon laser, a fast photodetector with the time resolution $20 \mu \mathrm{s}$, and a digital oscilloscope with the frequency $200 \mathrm{MHz}$ A system of mirrors 10,11 and light filters at the input of photodetector 12 were used to exclude the contribution from high-power pulsed radiation of a Nd:YAG-laser into the experimental results due to spatial and spectral separation of the diffracted beam and of the pulsed beams forming a hologram.

The characteristic oscillograms of diffracted radiation are shown in Fig. 6. A detailed analysis of the oscillograms at different time scales reveals two formation mechanisms of dynamic holograms with the relaxation times differing by three orders of magnitude. For relatively low intensities, about $1 \mathrm{MW} / \mathrm{cm}^{2}$, relaxation time of the diffracted signal is at a level of several seconds. For the intensities above $5 \mathrm{MW} / \mathrm{cm}^{2}$, relaxation time of the emerging fast component is at a level of milliseconds. A contribution of the fast dynamic-hologram recording mechanism is dominant

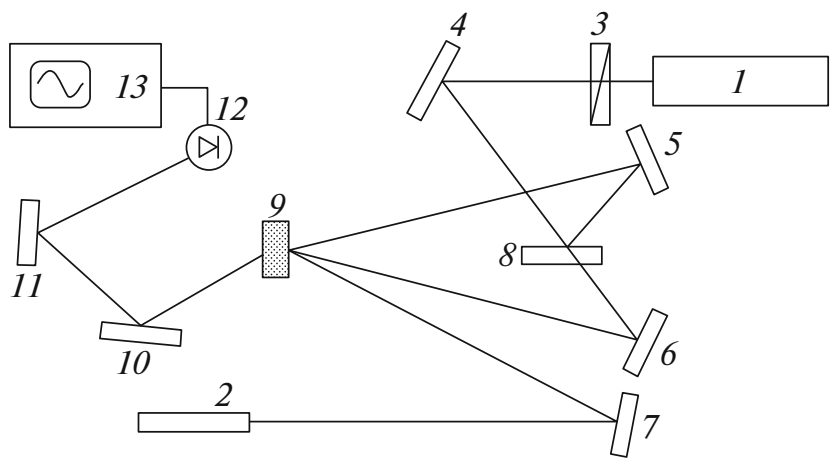

Fig. 5. The experimental setup, where 1-yttrium aluminum garnet laser; 2-helium-neon laser; 3-half-wave plate, 4-8, 10, 11- mirrors; 9-bismuth silicate crystal; 12-fast photodetector; 13-digital oscilloscope.

for high intensities of laser radiation exceeding 10$15 \mathrm{MW} / \mathrm{cm}^{2}$. This pattern is illustrated in Fig. 7 presenting the curves for the formation time of a slow grating component (a) and for the amplitude ratio of slow and fast components of a dynamic grating (b) as a function of the intensity of hologram recording laser radiation.

As demonstrated by a quantitative analysis of the relaxation dynamics of the dynamic-grating slow and fast components, the approximation is adequate when using a formula, similar to (14), with separation of the diffracted signal into two components. Figure 8 gives the curves for relaxation time of the dynamic-grating fast and slow components as a function of the hologram recording laser radiation. It is seen that relaxation times of a slow component come to several seconds. And characteristic times of the fast component emerging at the intensities above $5 \mathrm{MW} / \mathrm{cm}^{2}$ come to milliseconds. It should be noted that at high intensities a significant contribution is made by the fast component at a level of $100 \mu \mathrm{s}$.
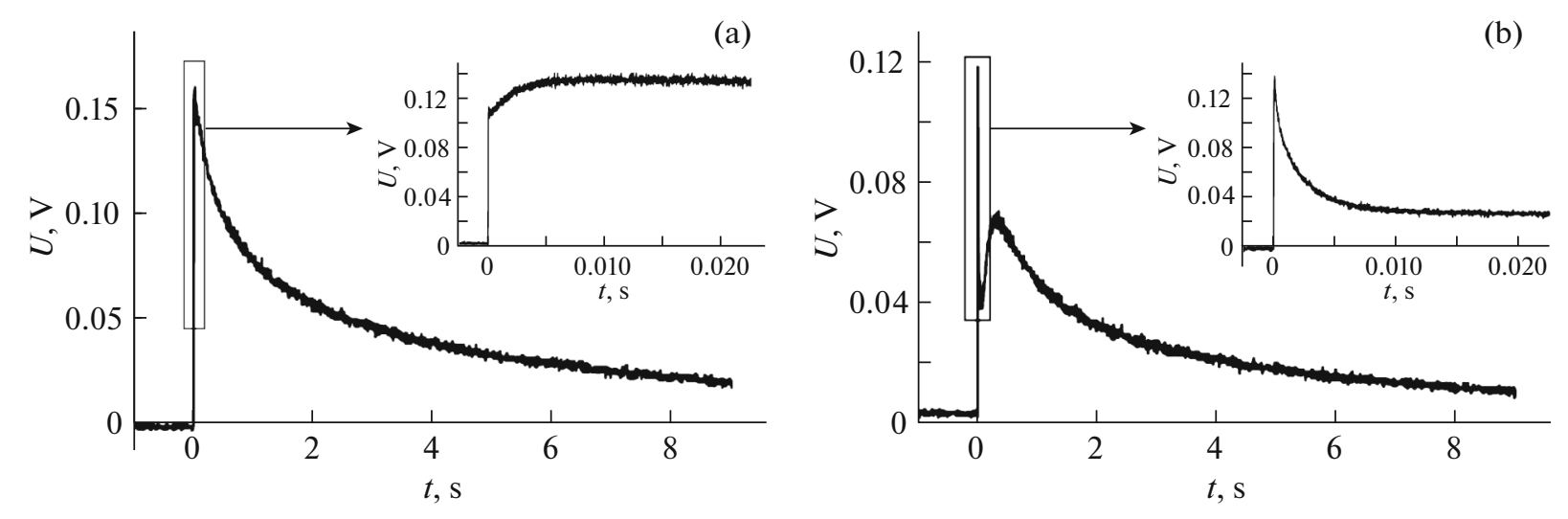

Fig. 6. Oscillograms of a diffracted signal when the intensities of laser radiation are 3 (a) and $15 \mathrm{MW} / \mathrm{cm}^{2}$ (b). 

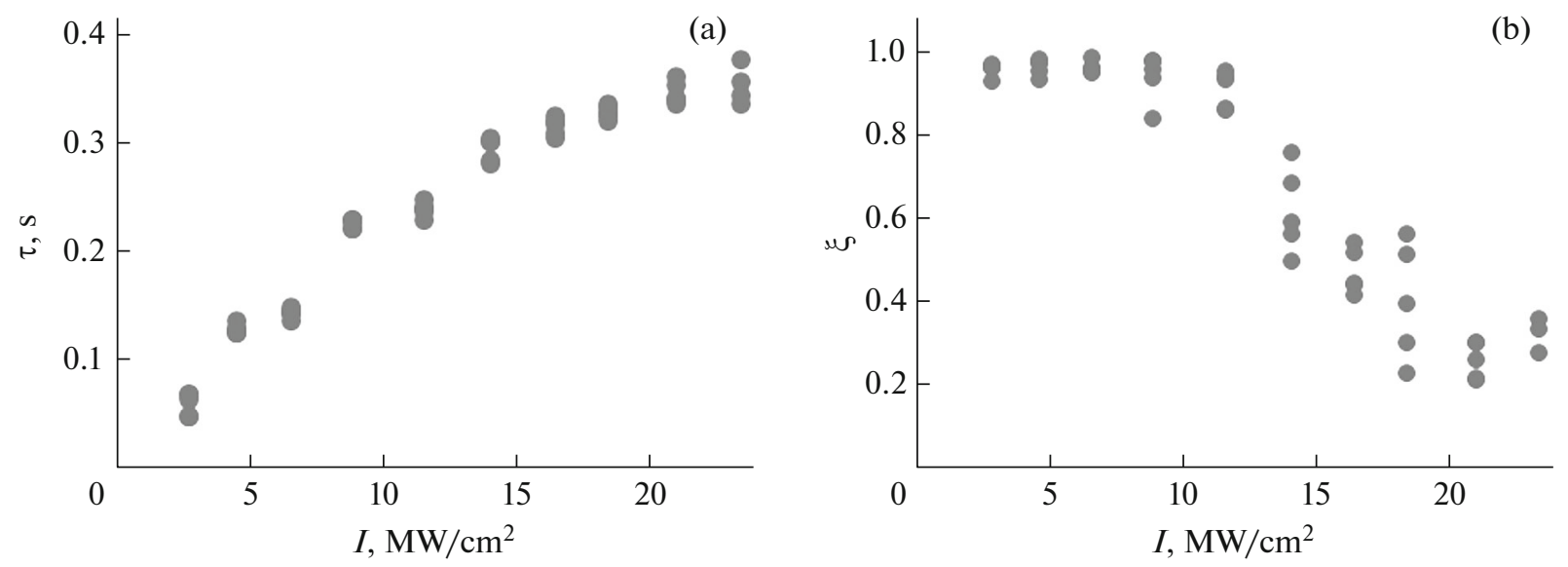

Fig. 7. The formation time of a long-lived grating (a) and the factor characterizing the amplitude ratio for slow and fast components of a dynamic grating (b) as a function of the intensity of laser radiation.
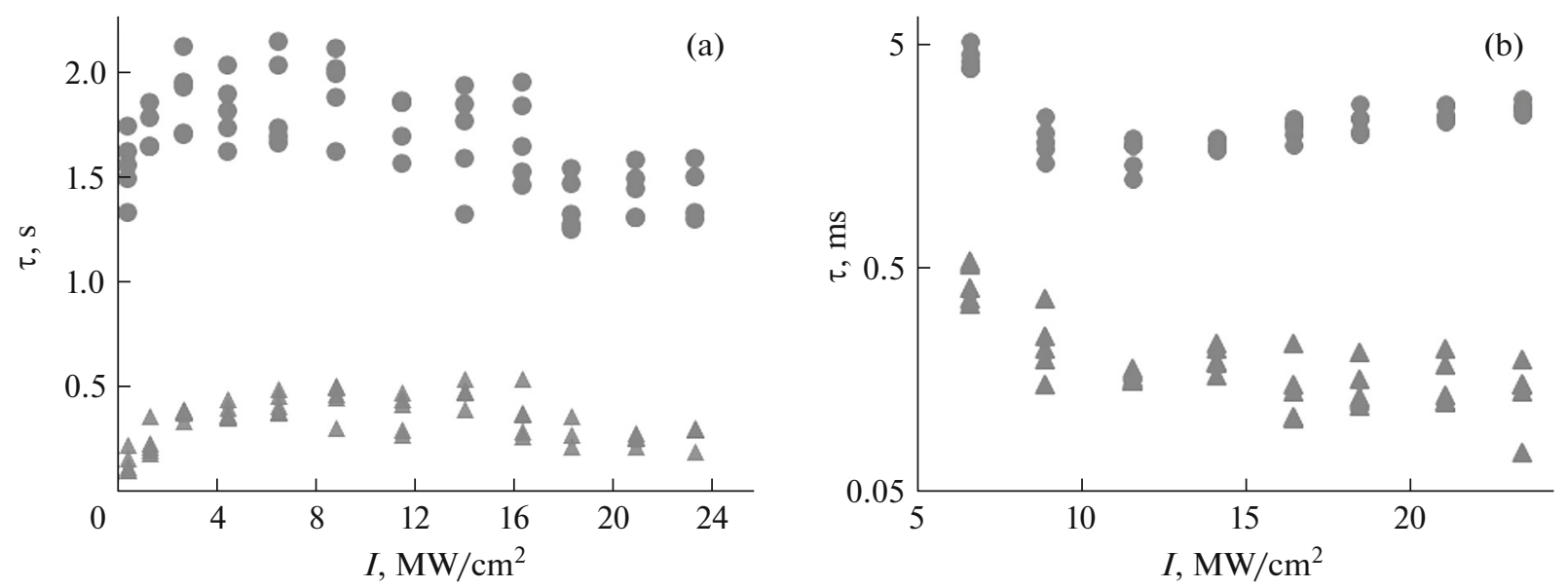

Fig. 8. The relaxation time of fast and slow components for a dynamic grating as a function of the intensity of hologram-recording laser radiation.

\section{CONCLUSIONS}

In this way, the paper presents an experimental study of the dynamics of photoinduced absorption and dynamic hologram recording in bismuth silicate crystals by high-power laser pulses with the length $20 \mathrm{~ns}$ at the wavelength $532 \mathrm{~nm}$. It is demonstrated that induced absorption takes an appreciable effect at the intensities above $1 \mathrm{MW} / \mathrm{cm}^{2}$, when there is population of the short-lived trapping levels, which have relaxation times of a few milliseconds or several tens of milliseconds. Dynamic hologram recording in these conditions has been realized; the possibility to form holographic gratings at a level of both milliseconds and seconds has been demonstrated. Based on comparison of relaxation dynamics for photoinduced absorption and holographic gratings, it is inferred that a fast formation mechanism of holographic gratings is associated with population of the short-lived trapping levels, which have millisecond relaxation times. The second mechanism of grating recording with relaxation times of several seconds is due to the formation of photorefractive nonlinearity in a field of the space charge. It has been found that, at high intensities, population involves the short-lived traps hindering diffusion of electrons in the conduction band and the formation of a space charge associated with the long-lived trapping levels.

\section{REFERENCES}

1. M. P. Petrov, S. I. Stepanov, and A. V. Khomenko, Photorefractive Crystals in Coherent Optics (Nauka, St. Petersburg, 1992) [in Russian].

2. D. C. Jones, S. F. Lyuksyutov, and L. Solymar, Appl. Phys. B 52, 173 (1991).

https://doi.org/10.1007/bf00750947

3. K. Buse, Appl. Phys. B 64, 273 (1997). https://doi.org/10.1007/s003400050175 
4. N. I. Nazhestkina, A. A. Kamsyilin, O. V. Kobozev, and V. V. Prokofiev, Appl. Phys. B 72, 767 (2001).

5. Yu. F. Kargin, V. I. Burkov, A. A. Mar'in, and A. V. Egoryshev, $\mathrm{Bi}_{12} \mathrm{Si}_{x} \mathrm{O}_{20-\delta}$ Crystals with Sillenite Structure. Synthesis, Structure, Properties (IOKhN RAN, Moscow, 2004) [in Russian].

6. S. M. Shandarov, N. I. Burimov, Yu. N. Kul'chin, R. V. Romashko, A. L. Tolstik, and V. V. Shepelevich, Quantum Electron. 38, 1059 (2008). https://doi.org/10.1070/qe2008v038n11abeh013793

7. Pochi Yeh, Introduction to Photorefractive Nonlinear Optics (Wiley, New York, 1993).

8. S. Wevering, J. Imbrock, and E. Kratzig, J. Opt. Soc. Am. B 18, 472 (2001).

https://doi.org/10.1364/josab.18.000472

9. A. Matusevich, A. Tolstik, M. Kisteneva, S. Shandarov, V. Matusevich, A. Kiessling, and R. Kowarschik, Appl. Phys. B 92, 219 (2008).

https://doi.org/10.1007/s00340-008-3098-z

10. A. Matusevich, A. Tolstik, M. Kisteneva, S. Shandarov, V. Matusevich, A. Kiessling, and R. Kowarschik, Appl. Phys. B 96, 119 (2009).

https://doi.org/10.1007/s00340-009-3512-1

11. M. G. Kisteneva, A. S. Akrestina, D. O. Sivun, R. V. Kiselev, S. M. Shandarov, S. V. Smirnov, A. L. Tolstik, I. N. Agishev, A. V. Stankevich, and
Yu. F. Kargin, Dokl. Tomsk.Univ., Sist. Upravl. Radioelektron. 22 (2), 62 (2010).

12. E. S. Khudyakova, M. G. Kisteneva, S. M. Shandarov, T. A. Kornienko, A. L. Tolstik, and Yu. F. Kargin, Radiophys. Quantum Electron. 57, 589 (2015). https://doi.org/10.1007/s11141-015-9543-z

13. T. Kornienko, M. Kisteneva, S. Shandarov, and A. Tolstik, Phys. Proc. 86, 105 (2017). doi j.phpro.2017.01.029

14. A. L. Tolstik, A. Yu. Matusevich, M. G. Kisteneva, S. M. Shandarov, S. I. Itkin, A. E. Mandel', Yu. F. Kargin, Yu. N. Kul'chin, and R. V. Romashko, Quantum Electron. 37, 1027 (2007). https://doi.org/10.1070/qe2007v037n11abeh013371

15. J. P. Hermann, J. P. Herriau, and J. P. Huignard, Appl. Opt. 20, 2173 (1981). https://doi.org/10.1364/AO.20.002173

16. J. P. Partanen, P. Nouchi, J. M. C. Jonathan, and R. W. Hellwarth, Phys. Rev. B 44, 1487 (1991). https://doi.org/10.1103/physrevb.44.1487

17. J. G. Murillo, Opt. Commun. 159, 293 (1999). https://doi.org/10.1016/s0030-4018(98)00610-5

18. A. V. Stankevich, A. L. Tolstik, and H. K. Haider, Tech. Phys. Lett. 37, 746 (2011). https://doi.org/10.1134/s1063785011080268

19. A. L. Tolstik and Kh. K. Khanon, Vestn. Belorus. Univ., Ser. 1: Fiz. Mat. Inform., No. 2, 3 (2012). 